\title{
Stationary Solid Particle Attractors in Standing Waves
}

\author{
Marcello Lappa ${ }^{1}$
}

${ }^{1}$ Telespazio, Via Gianturco 31 - Napoli, 80046 - Italy, marlappa@unina.it,marcello.lappa@telespazio.com

\begin{abstract}
The present analysis extends earlier theories on patterns formed by the spontaneous accumulation and ordering of solid particles in certain types of flow by considering the case in which the particle carrier flow has the typical features of a "standing wave". For the first time an explanation for this phenomenon is elaborated through arguments based on the interplay between vorticity and wave-interference dynamics (following a deductive approach after the so-called phaselocking or "resonance" model originally introduced by Pushkin et al., Phys. Rev. Lett., 106, 234501 (2011) and later variants developed by Lappa, Phys. Fluids, 25, 012101, (2013) and Chaos, 23, 013105, (2013)). The results of dedicated numerical simulations are used in synergy with available experimental work. An interesting analogy is proposed with the famous Chladni's series of experiments on patterns formed by sand on vibrating plates.
\end{abstract}

\section{INTRODUCTION}

Two-phase dispersive flows are found under many natural and technical conditions. The relevance of such phenomena is wide ranging and touches various scientific disciplines, including (but not limited to) environmental sciences, thermal and materials engineering, crystal growth, biology and bioengineering, and many other modern applications primarily driven by microtechnology. The spatio-temporal behaviour of the dispersed phase (immiscible "impurities" of different types, e.g., small drops, bubbles, solid or even "elastic" pieces of matter such as biological cells, hereafter simply and generically referred to as "particles") has become over the years a subject of great interest (from both practical and theoretical points of view).

In general, it is known that the related dynamics can be characterized in the phase space (a multidimensional phase space whose dimension corresponds to the number of degrees of freedom of the considered dynamical system) in terms of "attractors". Indeed, a well-known characteristic of dissipative systems is that their phase trajectories are attracted by special sets of points (having the property that different trajectories, arising from them, fall on them anyway, from which their denomination of "attractors"). The nature and "multiplicity" of these geometric objects depend essentially on the specific dynamics considered.

For the case of particles transported by a carrier flow, this subject becomes even more interesting and aesthetically appealing as the existence of attractors in the phase space is fully reflected in the formation of coherent particulate structures in the physical space (the abovementioned attractors exist in the full phase space as invariant sets, but what we observe in practice is always a projection of these sets onto the physical space).

It is known that particle relocation in a liquid (particles completely removed from specific regions of the domain) can be induced by a variety of effects (gravity, surface tension, shear, electric or magnetic fields $)^{1-9}$. As an example, in the Earth's gravitational field the usual differences in density cause rapid spatial segregation of particles through sedimentation or flotation; in the absence of 
gravity (microgravity) similar phenomena can be induced by gradients of surface tension. In technological casting applications all these effects typically prevent a homogeneous distribution of particles in the melt, hence limiting the industrial exploitation of many alloys. There is a variety of additional instances that could be cited. The microstructure of several immiscible blend systems depends strongly on the events that occur during processing. As recently illustrated by Saeedi et al. ${ }^{10}$, moreover, all these phenomena and related studies are also relevant in many cases to nanotechnologies and related products. Self-assembly is emerging as one of the main methods for construction of heterogeneous systems consisting of multiple component types in nano- and microscales. What is even more remarkable in such a field, microfabrication has opened the door to new ground-breaking concepts for phase separation, such as "deterministic hydrodynamics", by which distinct (solid/liquid) phases can be controlled on the basis of innovative ideas taking advantage of the intrinsic properties of the considered fluid-dynamic systems (related bifurcations, patterns and more or less pronounced degree of turbulence).

In particular, a very interesting and fruitful line of inquiry, which does not rely on a specific type of force or flow and is, therefore, applicable to a variety of cases and situations, is that related to the spontaneous particle association promoted by inertia (i.e. by small deviations induced on particle trajectories by their finite mass and volume with respect to the paths that would be ideally followed by weightless and sizeless tracers, Michaelides ${ }^{11}$; Balkovsky et al. ${ }^{12}$; Benczik et al. ${ }^{13}$ ).

Some interesting criteria to predict the inertial particle relocation/clustering dynamics in threedimensional steady or two-dimensional time-periodic flows were elaborated by Sapsis and Haller ${ }^{14}$. In these studies the particles were found to be attracted by inertial Lagrangian coherent structures (ILCS) that are smooth deformations of invariant tori.

In such a context, over recent years, several investigators (see the excellent studies by Schwabe et al. ${ }^{15-16}$ and Ueno et al. ${ }^{17-18}$ ) have reported on the formation of patterns given by the aggregation of small particles in three-dimensional incompressible model flows consisting of one or more rolls with toroidal structure and a travelling wave (TW), i.e. a disturbance travelling in the azimuthal direction.

On the one hand, related particle accumulation structures (PAS) have been frequently observed in experiments. On the other hand, however, theoretical research conducted by different groups has shown little agreement. Indeed, there is no unified theory yet for this phenomenon (the reader is referred to, e.g., Kuhlmann et al. ${ }^{19}$ for a recent review of a large cross section of fundamental research on this topic). Schwabe et al. ${ }^{16}$ originally interpreted this process in terms of a "resonance" mechanism. While Pushkin et al. ${ }^{20}$ introduced later the related concept of "phase locking" somehow resembling that elaborated by other authors ${ }^{21-23}$ to explain a fascinating companion phenomenon (suspended particles in a two-dimensional periodic lattice of obstacles following periodic trajectories which exhibit directional locking into lattice directions), Muldoon and Kuhlmann ${ }^{24}$ described it in terms of a structural instability of an elliptic orbit of a Kolmogorov-Arnold-Moser (KAM) torus (which is an time-invariant topological feature of the flow). Moreover, even the pertinence of the description in terms of supercritical Marangoni flow, as the sole paradigmatic precondition able to produce this phenomenon, is still a debated point ${ }^{25}$.

In the first case (Schwabe et al et al. ${ }^{16}$, Pushkin et al. ${ }^{20}$ ), the resonant (inertial) coupling between travelling fluid-dynamic disturbances and the typical particle turnover time (the characteristic time taken by the particle to follow a given closed path) is at the root of the theory. A later revisitation of this model in terms of particle axial vorticity is due to Lappa ${ }^{26}$, who replaced the particle turnover 
frequency with equivalent arguments on the particle angular spin. This variant has its basis in the ideas originally developed (although in a completely different theoretical environment) by Rhines ${ }^{27}$, who identified a process able to support the formation of azimuthally elongated structures in atmospheric flows at the scale where the period of atmospheric (Rossby) waves matches the turnover time of typical eddies (vortices) associated with atmospheric turbulence.

In this study, we wish to show that this class of (inertial) models, can become effective also in explaining the bizarre phenomena which emerge when the considered flow has the form of a standing wave (SW), provided it is properly reinterpreted and extended.

As shown by recent experiments (Fig. 1a, Schwabe and Mizev ${ }^{28}$ ), for which an explanation has not been found yet, when the flow is a standing wave, the patterns created by the spontaneous demixing and accumulation of particles show an appearance that is totally different from the PAS formed by travelling waves. The essentially stationary nature of the resulting pattern as seen in the laboratory frame and its topological properties distinguish such a case from PAS phenomena observed previously (for which particles were seen to collect into a seemingly rigid closed path rotating in the laboratory frame at the same angular frequency of the travelling wave so as to give the illusion of a stationary solid circuit in the reference frame rotating with the wave).

It is unknown whether the mechanisms supposed to be operative in the case of TW still play a role in this case or not. From available experiments (Fig. 1a), one can see that the particle pattern consists of $2 \mathrm{~m}$ sectors ( 6 sectors for $\mathrm{m}=3$ where $\mathrm{m}$ is the azimuthal wavenumber), which, like 6 pieces of a cake, are separated by 6 sharp radial stripes formed by close particles ${ }^{28}$. The pattern also includes an inner particle circuit with shape and average radial position varying in time (a snapshot of this circuit is sketched for clarity in Fig. 1b).

It is shown here how these new intriguing dynamics, which can be explained neither in terms of stagnation surfaces (the aforementioned radial branches and the inner circuit are not stagnation loci), nor on the basis of KAM tori (given the absence in the considered flow of azimuthally extended closed stable streamtubes, whose existence is a necessary prerequisite of that class of models) can be given a relatively simple explanation if the problem is cast in the form of axial vorticity dynamics and related physical connections with inertial mechanisms.

\section{MATHEMATICAL MODEL}

\section{A. The Pulsating state and the vorticity-wave interaction mechanism}

To fully understand the consequences of the above (axial-vorticity-dynamics based) way of thinking (and appreciate the related theoretical implications for the considered problem), we have to recall shortly the general properties of a standing wave (the reader is referred to, e.g., Shevtsova et al. ${ }^{29}$ for the companion regime in which the flow has the spatio-temporal structure of a TW). In physics, a standing wave (also known as a stationary wave), is a wave (a disturbance oscillating in time) that remains in a constant position. In general, in a stationary medium this phenomenon emerges as a result of the interference (superposition) of two waves having the same amplitude and same angular frequency $\omega$, but travelling in opposite directions. This typically results in a field with no net disturbance transport on average along the propagation direction of the two component waves (the azimuthal direction $\varphi$ in the present case for which we consider a liquid bridge). In terms of 
patterning behaviour, the final effect is a series of nodes (disturbance zero amplitude) and antinodes (disturbance maximum amplitude) at fixed locations along $\varphi$ (as shown, e.g., by Lappa et al. ${ }^{30}$, the node/anti-node distance is $\lambda / 2$ where $\lambda=\pi / \mathrm{m}$ ). Albeit there is no net disturbance transport along the positive (or negative) azimuthal direction, the disturbance propagates from each anti-node to the two surrounding nodes with variable (location-depending) angular velocity. More specifically, by indicating with $\Omega$ the variable disturbance angular frequency, the following conditions represent a good phenomenological model of a SW: $\Omega=0$ on all nodes, $|\Omega|=\omega$ on all antinodes, with all points located in other meridian planes experiencing an apparent disturbance angular velocity $0<\Omega \leq \omega$ (Fig. $1 b)$.

The above description can be applied to both thermal and velocity (or vorticity) disturbances. In the following, we will expressly concentrate on axial vorticity $[\underline{\nabla} \wedge \underline{V}]_{z}$ as the wave representative disturbance (where $\underline{V}$ is the fluid velocity and the subscript $\mathrm{z}$ denotes the component of the curl operator in the axial direction; as shown by Lappa ${ }^{26}$, this quantity assumes a particularly meaningful role, as it is everywhere equal to zero in the axisymmetric (steady) state that precedes the onset of three-dimensional (3D) flow and assumes values $\neq 0$ in the supercritical state only, where, more specifically, its half can be regarded as a measure of the local average angular velocity (spin) of the considered fluid element about the vertical direction).

Before discussing the detailed results of specific calculations, it is helpful to consider some general physical consequences of the above representation of SW when it is considered in combination with the resonance (phase-locking) theory. In the light of such a theory and related revisitation provided by Lappa (hereafter, simply referred to as the vorticity-wave model), particles should accumulate where the angular spin

$\Theta_{\text {fluid }}=\frac{1}{2}[\underline{\nabla} \wedge \underline{V}]_{z}$

attains a value $\cong 0$. Any particle located at a position where $\Theta_{\text {fluid }} \neq 0$ and, in particular, $\Theta_{\text {fluid }} \cong \Omega$, in fact, would tend to undergo the vorticity-wave coupling/locking mechanism and, as a result, to leave its initial position (moving in the azimuthal direction by virtue of the resonance (or synchronization) physical process initially theorized by Schwabe et al. ${ }^{16}$ ).

\section{B. Nondimensional field equations}

To verify these arguments, here, the Navier-Stokes and energy equations have been solved numerically in cylindrical coordinates $(r, z, \varphi)$. The related nondimensional form has been obtained using conventional thermal scalings ${ }^{26}$ (i.e. the axial system extension (L) as a reference length, the energy diffusion velocity $\mathrm{V}_{\alpha}=\alpha / \mathrm{L}$ as a reference velocity and all the other reference quantities derived accordingly). The resulting equations read:

$\underline{\nabla} \cdot \underline{\mathrm{V}}=0$

$$
\frac{\partial \underline{V}}{\partial}=-\underline{\nabla} p-\underline{\nabla} \cdot[\underline{V} \underline{V}]+\operatorname{Pr} \nabla^{2} \underline{V}
$$




$$
\frac{\partial T}{\partial t}+\underline{\nabla} \cdot[V T]=\nabla^{2} T
$$

where $\operatorname{Pr}=v / \alpha$ (kinematic viscosity/thermal diffusivity) is the well-known Prandtl number.

To track particle motion we have used the Maxey-Riley equation (see, e.g., Babiano et al. ${ }^{31}$ ):

$$
\frac{d \underline{V}_{\text {partcl }}}{d t}=\frac{1}{\xi+1 / 2}\left[-\frac{\operatorname{Pr}}{S t}\left(\underline{V}_{\text {partcl }}-\underline{V}\right)+\frac{3}{2} \frac{D \underline{V}}{D t}\right]
$$

where $\underline{V}_{\text {part }}$ and $\underline{V}$ are the particle and fluid velocity, respectively (the velocity components in the axial, radial and azimuthal directions being denoted by $V_{\mathrm{z}}, V_{\mathrm{r}}$ and $V_{\varphi}$, respectively). Moreover, $\xi$ is the ratio of the particle to the fluid density and $\mathrm{St}$ is the Stokes number defined as

$$
S t=\frac{\tilde{\tau} \nu}{L^{2}}=\frac{\eta}{\xi-1} \operatorname{Pr}
$$

where $\widetilde{\tau}$ is the so-called relaxation time, linked to the solid particle radius $\widetilde{R}$ by the expression

$$
\widetilde{\tau}=\frac{2}{9} \frac{\widetilde{R}^{2}}{v}
$$

and $\eta=\frac{\tilde{\tau} \alpha}{L^{2}}(\xi-1)$ is a general parameter ${ }^{26}$ accounting for the combined effect of particle inertia and drag $\left(\eta=\mathrm{O}\left(10^{-5}\right), \operatorname{Pr}=\mathrm{O}(10)\right.$ and $\xi=\mathrm{O}(1)$ for the conditions considered here $\left.\rightarrow \mathrm{St}<<\mathrm{O}(1)\right)$.

\section{The study case}

As a sample SW we use typical supercritical Marangoni flow, but conclusions are applicable to pulsating flows of gravitational nature as well (see, e.g., Boronska and Tuckermann ${ }^{32}$ ) and, in general, to any flow with the characteristics of a SW.

In particular, we focus on the following well known test case (Melnikov et al. ${ }^{33}$; Lappa ${ }^{26,34}$ ): a liquid bridge with aspect ratio $\mathrm{A}=0.34$ (A being the height to diameter ratio) delimited by solid horizontal disks (one at $\mathrm{z}=0$ cooled, the other at $\mathrm{z}=1$ heated, adiabatic conditions on the liquid/gas interface). The considered values of the Prandtl and Marangoni numbers are $\operatorname{Pr}=8\left(\mathrm{NaNO}_{3}\right)$ and $\mathrm{Ma}=2.06 \times 10^{4}$, respectively (Ma defined as $\sigma_{\mathrm{T}} \Delta \mathrm{TL} / \mu \alpha$ where $\sigma_{\mathrm{T}}$ is the surface tension derivative and $\mu$ the dynamic viscosity). Moreover, no steady residual gravity is considered $(\mathrm{Ra}=0)$ and no arbitrary disturbances are added to the basic state to accelerate the transition to 3D flow. For these conditions $\omega \cong 71.4$ and the supercritical state displays the same succession of stages of evolution already observed for highPr liquids in other circumstances ${ }^{30}$ (initial pattern with disturbances growing exponentially in time, followed by the transitory appearance of the standing wave, finally replaced by a rotating TW pattern as the asymptotic equilibrium waveform ${ }^{26,34}$ ). 
The value of $\eta$ is $1 \times 10^{-5}$, which corresponds to the particles with density (solid/fluid) ratio $\xi=1.85$ and size $45 \mu \mathrm{m}\left(\mathrm{St} \cong 10^{-4}\right)$ considered by Melnikov et al. $^{33}$. The numerical simulations with the dispersed solid phase have been limited to the timeframe with the oscillatory Marangoni flow behaving as a pure standing wave (temperature disturbances simply consisting of a number $\mathrm{m}$ of couples of spots (hot and cold) pulsating at fixed azimuthal positions along the interface with no net disturbance transport on average along the azimuthal direction and no net azimuthal flow). Before starting the numerical computations, solid particles $\left(\mathrm{N}_{\text {particles }}=10^{4}\right)$ have been seeded uniformly into the computational domain assuming the initial velocity of each particle equal the local fluid velocity.

\section{Solution method and numerical accuracy}

The mass, momentum, energy and the particle-motion equations (2-5) have been solved over a cylindrical mesh $\left(\mathrm{N}_{\mathrm{z}} \mathrm{XN}_{\mathrm{r}} \mathrm{xN}=32 \times 40 \times 40\right)$ with a time integration step $\Delta \mathrm{t} \cong 5 \times 10^{-8}$ (required for the stability of the used time-explicit primitive-variable algorithm). Specific details about these methods have been already published and are not duplicated here for the sake of brevity. For a more in-depth analysis the interested reader is referred to the original work (see, e.g., Refs ${ }^{35-37}$; while for a validation study of the overall fluid-solid phases tracking algorithm, the more recent $\mathrm{Ref}^{34}$ may be considered). Here we limit ourselves just to mentioning that eq. (5) does not account for a back influence of particles on convection (it is based on a one-way coupling assumption, i.e. particle motion cannot alter the liquid flow). Moreover, particles are assumed to be independent of each other (this is permissible if the concentration of the dispersed phase in the flow is small, i.e. if the distance of a particle from others is much larger than their characteristic size, see again $\operatorname{Ref}^{34}$ and references therein). Despite such approximations, however, tracers moving close to the solid boundaries require a special treatment (given the no-slip properties of such surfaces acting as sinks of momentum and, therefore, as potential particle-entrapping loci). Following earlier studies, in particular, we assume the dispersed solid phase to interact in a non-elastic fashion with walls (in practical numerical implementation, this means a particle can approach the solid boundary until a distance not smaller than its radius is achieved; then it is allowed to slide along the boundary until the wall-normal velocity component becomes directed inwards). The same condition has been implemented also for two limited portions ( $5 \%$ of L) of the free liquid/gas interface (located in proximity to both the cold and hot corners to avoid the unphysical accumulation of particles there noticed during the preliminary tests performed for the validation of the algorithm).

The underlying assumptions on which this approach is based are, therefore, particles with solid/fluid density ratio $\xi \neq 1$, a perfectly spherical shape, non-elastic particle-wall interaction and a very small value $(<<1)$ of the particle to fluid system characteristic size ratio (which implies a particle response time much less than the characteristic time scales of the considered flow, this condition being a necessary prerequisite for the validity of the Maxey-Riley equation ${ }^{34}$ ). For such conditions, the resulting numerical error related the integration of eq. (5) with a first-order scheme (accumulated over the number of iterations $\mathrm{N}_{\text {iter }}$ performed to study the phenomenon, see, e.g., Atkinson ${ }^{38}$ ) can be estimated as $\mathrm{N}_{\text {iter }} \Delta \mathrm{t}^{2}=\mathrm{O}\left(10^{-8}\right.$ ) (in the present case $\mathrm{N}_{\text {iter }}$ being $\cong 1.2 \times 10^{7}$ ). 


\section{RESULTS AND DISCUSSION}

As a confirmation of the arguments developed in the preceding text (see, in particular, Sect. II.A), the simulations have shown that, when the supercritical Marangoni flow is in the so-called pulsating (SW) regime, extended regions exist at any time and in each sector where $0<\left|\Theta_{\text {fluid }}\right| \leq \Omega_{\max }\left(\Omega_{\max }=\omega\right)$ and, therefore, resonance (vorticity-wave coupling) between particles and the travelling disturbance is expected to take place (Fig. 1c).
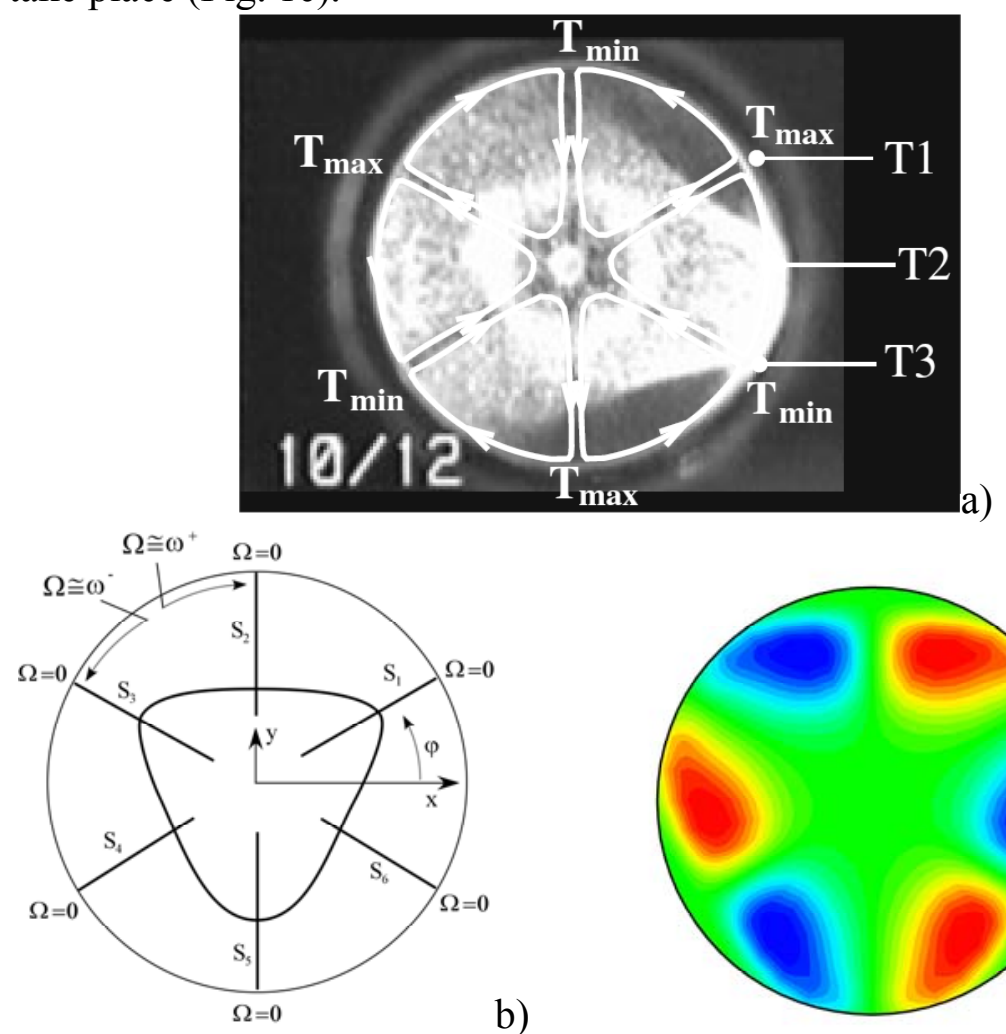

b)

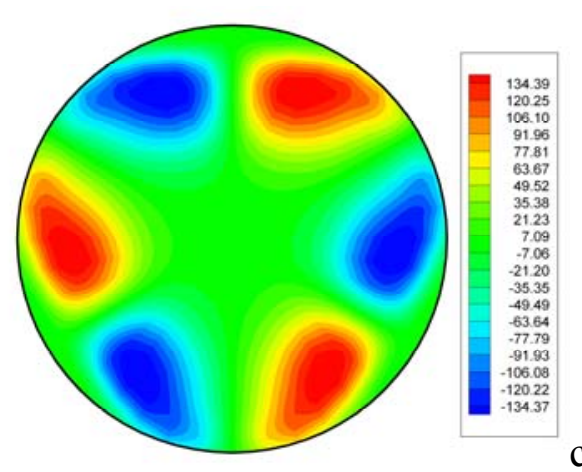

c)

Fig. 1: Standing PAS: a) Projection in the xy plane of particle distribution (experimental results, snapshot, courtesy of D. Schwabe and A. I. Mizev); b) related sketch showing the regions where particle preferential concentration occurs (given by 6 sharp radial stripes and an inner closed circuit having approximately a triangular shape); c) contour plot (numerical results, snapshot) of particle angular spin $\Theta_{\text {fluid }}=\left[\partial / \partial r\left(r V_{\varphi}\right)-\partial V_{r} / \partial \varphi\right] / 2 r$ at $\mathrm{z}=0.5$.

The numerical results (Figs. 2 and 3) also prove that, as expected, the particles accumulate exactly on the isosurfaces of $\Theta_{\text {fluid }}$ such that $\Theta_{\text {fluid }} \cong 0$. In very good agreement with experimental results, particles seem to "decorate" the boundaries between the 6 existing sectors.

Indeed, these are the only possible equilibrium positions. Any other location in space would be unstable with respect to the vorticity-wave mechanism. A particle leaving the surfaces $\Theta_{\text {fluid }} \cong 0$ would undergo the phase-locking process and finally move back to positions where $\Theta_{\text {fluid }} \cong 0$ (Figs. 4). Most interestingly, the numerical results also lead to the conclusion that the loci of points where $\Theta_{\text {fluid }}=0$ are not limited to the boundaries between the six aforementioned sectors. An isosurface extended over $360^{\circ}$ with $\Theta_{\text {fluid }}=0$, in fact, exists, whose radial position changes in time: it "pulsates" moving from the external surface (Fig. 3a) towards the axis of the liquid bridge (Fig. 3f) and vice versa. As evident in Figs. 3, particles stay also attached to such a surface; which one side provides further evidence to the validity of the present "equilibrium" model and, on the other side, explains almost perfectly the existence of the pulsating inner triangular particle circuit visible in Fig. 1. 


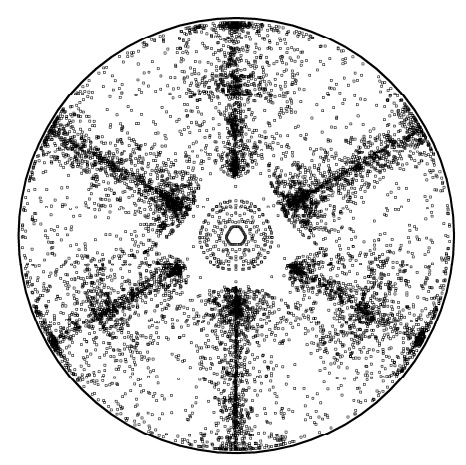

a)
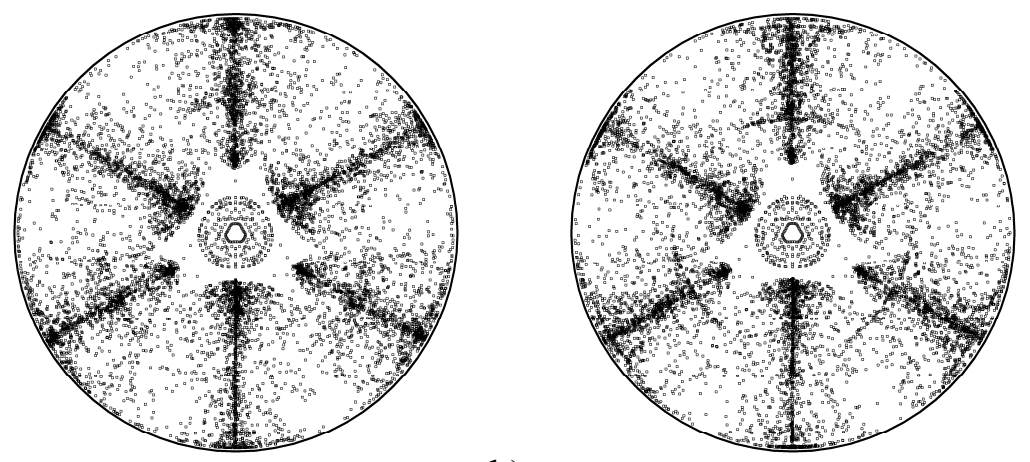

b)

c)
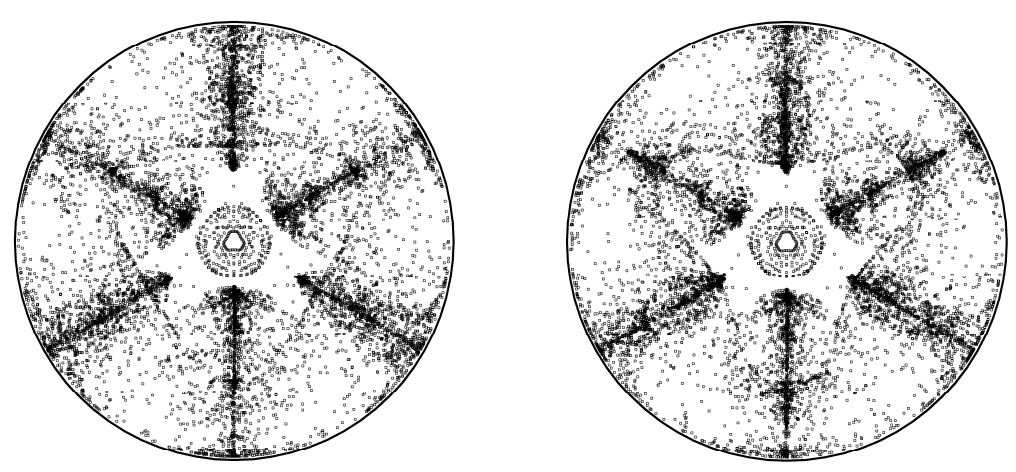

d)

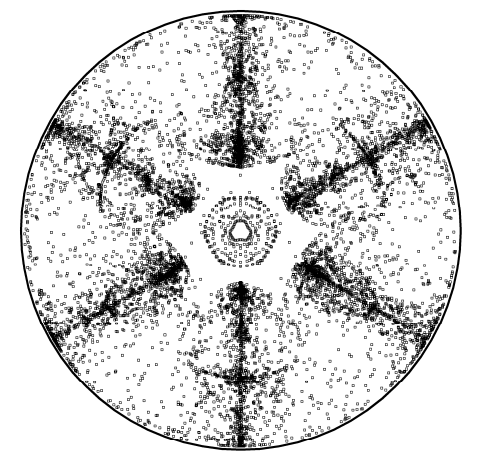

e)

Fig. 2: Snapshots (projection in the xy plane of the effective threedimensional particle distribution) equally spaced in time during half a period of oscillation $\tau$ (liquid bridge of $\mathrm{NaNO}_{3}, \operatorname{Pr}=8, \mathrm{~A}=0.34$, $\mathrm{Ma}=20600$, standing wave, $\tau=2 \pi / \omega, \omega=71.4, \xi=1.85$, St $\left.\cong 10^{-4}\right)$.
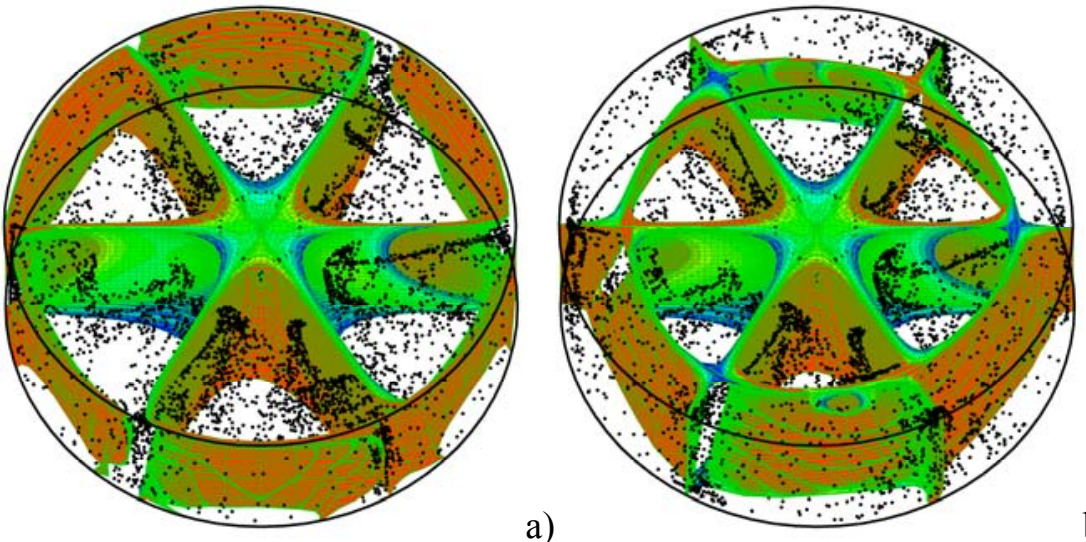

a)

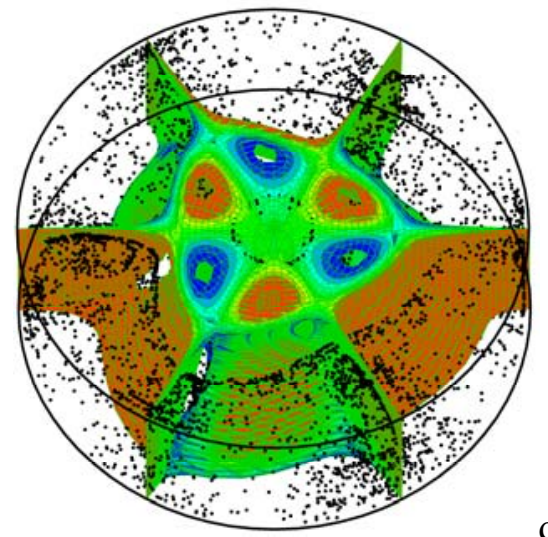

d)

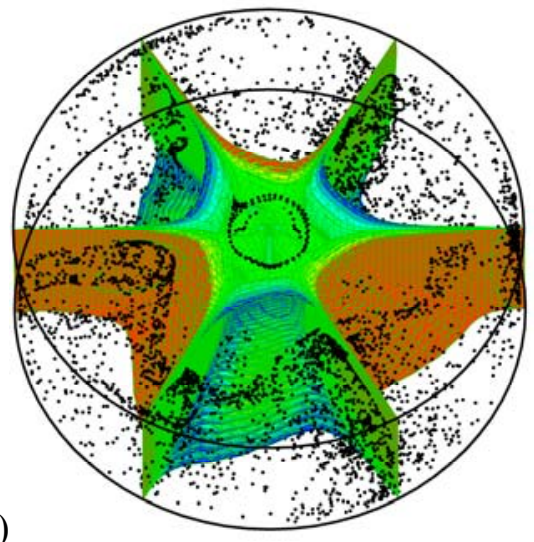

b)

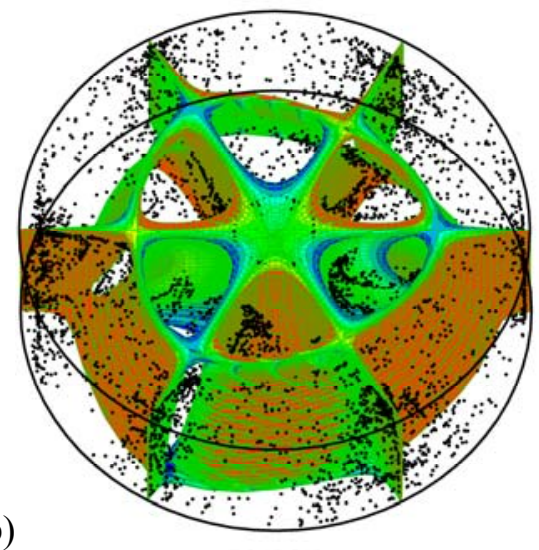

c)

Fig. 3: 3D snapshots (equally spaced in time) of particle distribution and isosurfaces of $\Theta_{\text {fluid }} \cong 0$ $\left(-1 \leq \Theta_{\text {fluid }} \leq 1\right)$ during half a period of oscillation $\tau$ (liquid bridge of $\mathrm{NaNO}_{3}, \operatorname{Pr}=8, \mathrm{~A}=0.34$, $\mathrm{Ma}=20600$, standing wave, $\tau=2 \pi / \omega, \omega=71.4, \xi=1.85$, St $\cong 10^{-4}, \mathrm{~N}_{\text {particles }}=10^{4}$ ). 
In this regard, it is worth pointing out a possible analogy with the famous phenomena originally discovered and studied by Ernst Chladni. This family of experiments (generally referred to as the "Chladni's acoustic experiments" ${ }^{39}$ ) has its basis in a plate or membrane covered in sand or similar material, that gets vibrated at various frequencies producing interesting patterns.

It is a well-known fact that a plate or membrane vibrating at resonance is divided into regions vibrating in opposite directions, bounded by lines of zero vibration called "nodal lines" (the loci of points where elastic waves produced in the plate by the application of external vibrations and propagating in different directions reunite and die). Chladni's technique consisted of drawing a bow over a piece of metal whose surface was lightly covered with sand. The plate was bowed until it reached resonance. At that stage, vibration was observed to cause the sand to move and concentrate along the nodal lines where the surface is still, thereby giving rise to various intricate patterns consisting of one-dimensional segments, curves and lines.

In view of such arguments, the affinity with present phenomena may be thus further elaborated as follows: Just as in the Chladni's experiments the sand initially bounces about on the plate until it settles in the areas of zero movement (thereby outlining the nodal lines which separate regions of the plate vibrating in opposite directions), the solid particles in the liquid bridge are pushed away from the areas of active hydrothermal disturbance propagation (the regions with $\Omega \neq 0$ where phaselocked particles tend to move in azimuthal direction via the vorticity-wave resonance process) and gather in the places where the combination of the two counterpropagating waves gives no effect (local disturbance amplitude and velocity zero).

Another possible way to justify the isomorphism between these two categories of phenomena simply consists of considering the possibility to replace formally:

- The two-dimensional space in the Chladni's experiments with the fully three-dimensional space in the present (liquid bridge) case,

- The "motion" effect exerted by resonant vertical plate vibrations on sand (via elastic coupling between sand beads and the underlying oscillating surface) with the motion of phase-locked tracers in azimuthal direction (via the vorticity-wave coupling mechanism),

- The loci of points where the plate remains motionless with the loci of points in the liquid bridges where the two counterpropagating waves combine such that the resulting disturbance has no amplitude and no velocity (i.e. $\Omega=0$ ). 

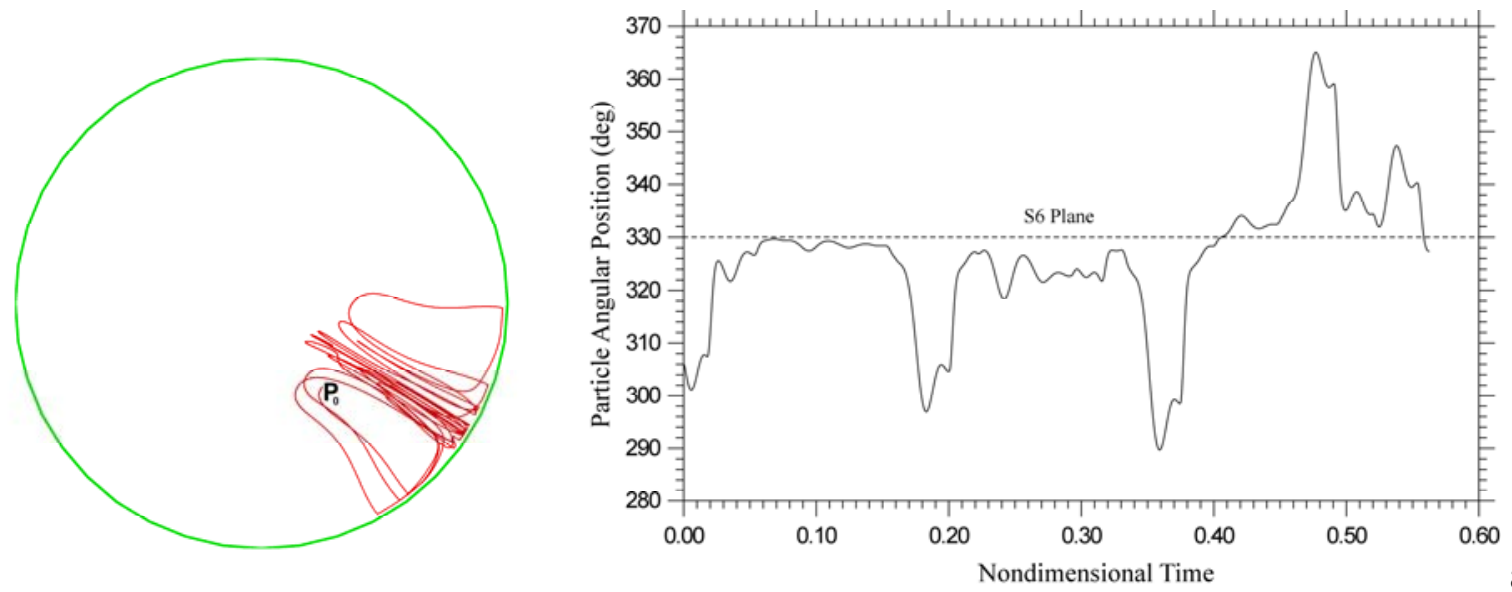

a)
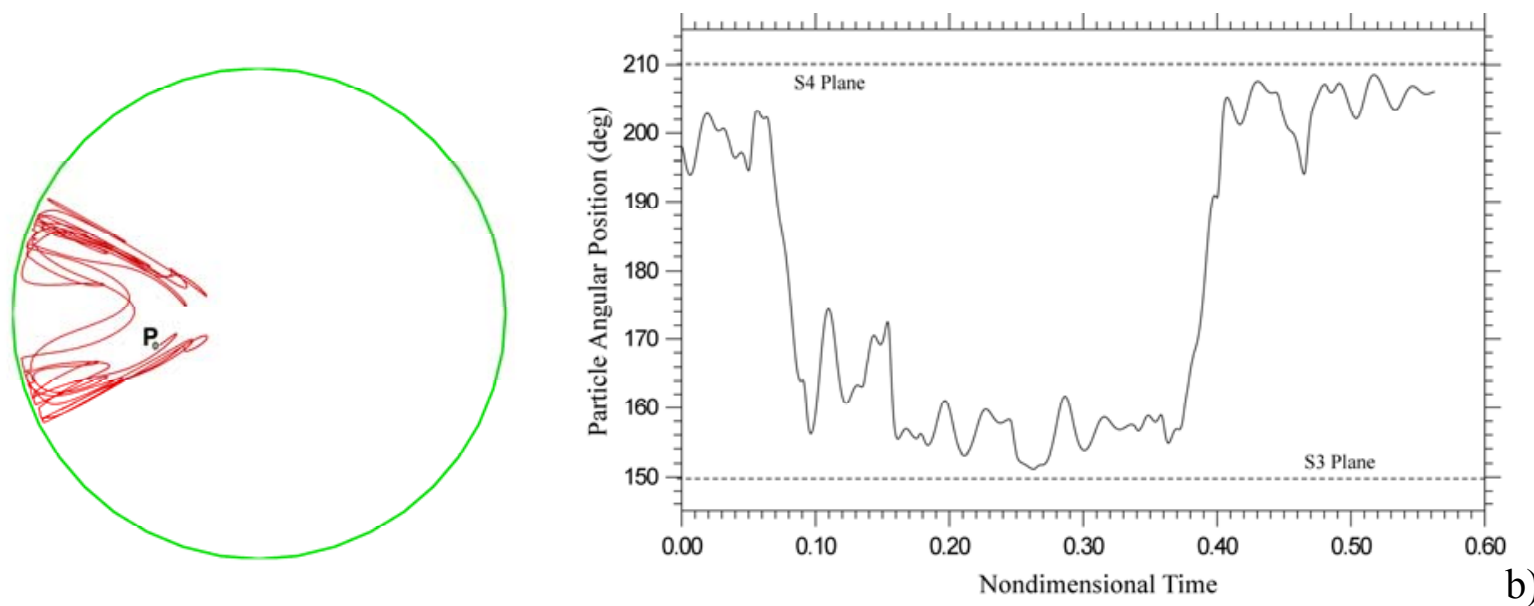

b)
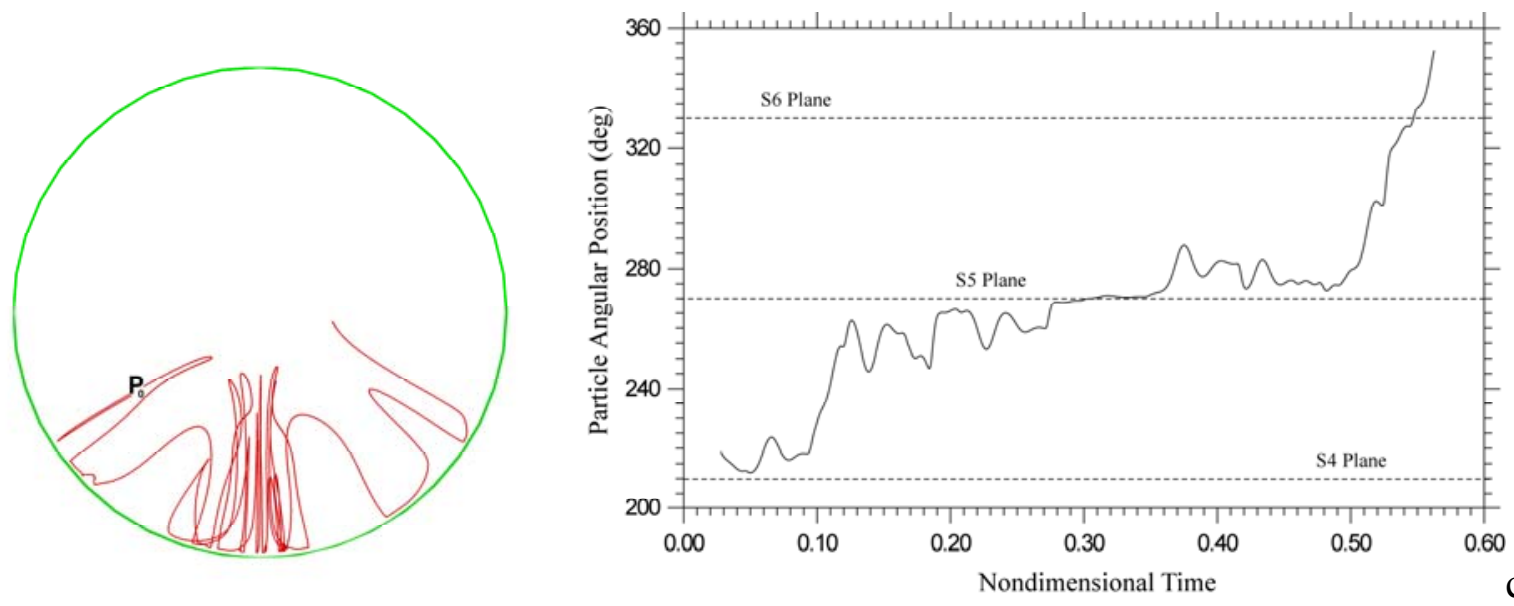

Fig. 4: Examples of typical particle trajectories (liquid bridge of $\mathrm{NaNO}_{3}, \mathrm{Pr}=8, \mathrm{~A}=0.34, \mathrm{Ma}=20600$, standing wave, $\xi=1.85, \mathrm{St} \cong 10^{-4}$, nondimensional time covered by the particle $0.56 \cong 6 \mathrm{x} \tau_{\text {wave }}$ where $\tau_{\text {wave }}=2 \pi / \omega, \omega=71.4$, initial particle position $\mathrm{P}_{0}=\left(\mathrm{r}_{0}, \varphi_{0}, \mathrm{z}_{0}\right)$ ): a) $\left.\mathrm{r}_{0}=0.62, \varphi_{0}=306^{\circ}, \mathrm{z}_{0}=0.597, \mathrm{~b}\right)$ $\left.\mathrm{r}_{0}=0.62, \varphi_{0}=198^{\circ}, \mathrm{z}_{0}=0.339, \mathrm{c}\right) \mathrm{r}_{0}=0.9, \varphi_{0}=213^{\circ}, \mathrm{z}_{0}=0.585$. 
The efficiency of the vorticity-wave mechanism in promoting the accumulation of particles on disturbance-less surfaces is clearly shown in Figs. 4, which provides some examples about typical trajectories followed by particles in space over a relatively long representative time interval $\left(6 \mathrm{x} \tau_{\text {wave }}\right.$ where $\left.\tau_{\text {wave }}=2 \pi / \omega\right)$.

On the basis of these figures particle trajectories may be categorized into three main types:

1) Trajectories that, arising from a point in proximity to an equilibrium radial surface (meridian plane separating two sectors), end on the equilibrium surface anyway (after covering a limited angular neighbourhood, Fig. 4a);

2) Trajectories of particles that move from an equilibrium meridian plane to another and then back to the initial plane, spending a very limited time in the intermediate space (Fig. 4b);

3) Trajectories that cover two sectors, still spending, however, a relatively limited time in regions separating equilibrium conditions (Fig. 4c).

In the first case, a particle undergoes some back and forth azimuthal oscillations across the equilibrium (radial) section (the S6 plane in Fig. 4a); the angular amplitude covered by the particle before returning to the equilibrium section (after the particle leaves it moving in the clockwise or anticlockwise direction) is approximately $\pi / 2 \mathrm{~m}$. The overall time spent by the particle in regions exceeding $10^{\circ}$ of angular distance from the equilibrium meridian plane is on average (over the monitored temporal extension $) \cong 25 \%$ of the considered timeframe $\left(6 \mathrm{x} \tau_{\text {wave }}\right)$.

In the second case, a particle escapes an equilibrium surface after performing several stable revolutions (complete orbits around the Marangoni toroidal vortex at an approximately constant azimuthal location) and rapidly moves to another equilibrium meridian plane (e.g., from the S4 to the S3 plane in Fig. 4b). After reaching the new equilibrium section, then the particle stays there for some time (again, covering several complete orbits) and finally it comes back to its starting surface. For such a case, the overall angular extension covered by the particle is obviously equal to the angular amplitude of a sector, i.e., $\pi / \mathrm{m}$, while the overall time spent in the space between the two bounding equilibrium meridian planes is only $\cong 15 \%$ of the considered timeframe $\left(6 \mathrm{x} \tau_{\text {wave }}\right)$.

The third case may be regarded as a variant of the second class of trajectories for which an incoming particle captured by an equilibrium surface (the S5 plane in Fig. 4c), after covering several complete orbits is then released in the next sector, thereby retaining its original sense of azimuthal propagation (from the S4 towards the S6 plane); in such a case the particle covers an overall angular space $\cong 2 \pi / \mathrm{m}$ in the considered timeframe.

Similar observation and measurements performed over a representative set of 100 particles (uniformly distributed in the 3D space at the initial instant) have led to the conclusion that the time spent on average by particles in the "regions of influence" of equilibrium sections Si (here a region of influence is defined as the portion of 3D space originating from the generic Si radial section with an angular extension $\Delta \varphi=10^{\circ}$ in each angular direction) is more than $80 \%$ of the computed time.

Such a percentage may be regarded as a possible quantification of the efficiency of the vorticitywave coupling/resonance mechanism in keeping particles spatially confined (thereby forming the recognizable patterns observable both experimentally and numerically).

According to the present numerical simulations, such efficiency even increases when the standing wave is taken over (as time passes ${ }^{30,40}$ ) by the TW. Indeed, the percentage of scattered (i.e. not undergoing accumulation in precise loci) particles tends to zero as the SW is fully replaced by the 
TW. Moreover, the formation of recognizable structures in the standing wave regime takes a time higher ( $\cong 50 \%$ more) than that required when the prevailing waveform is a travelling wave.

The reason for such differences perhaps may be found in the different "nature" of the equilibrium conditions established for particles in the two regimes. It is reasonable to assume that, given the well-defined and stable coupling between the propagating disturbance (at a constant angular speed $\Omega=\omega$ ) and the motion of particles, guaranteed by the condition $\Theta_{\text {fluid }}=\omega=\operatorname{const}^{25-26}$, the TW may provide an "asymptotically stable" condition in terms of vorticity-wave locking (in the Lyapunov stability sense), whereas the SW is able to provide no more than a situation of simple Lyapunov stability (given the intrinsic "alternating" nature of the mechanism keeping particles confined in proximity to equilibrium surfaces).

\section{CONCLUSIONS}

The mechanism related to the formation of the so-called particle accumulation structures is still not clear, and there exists today, to our knowledge, no definitive theoretical and/or empirical model that can adequately characterise such phenomena in every circumstance. Motivated by this observational tide, we have tested the basic dynamical principles governing the so-called resonance model in a specific situation (standing wave) where excluded are any processes that depend on the existence of invariant properties (regular closed streamtubes) or a preferential direction of propagation of the thermofluid-dynamic disturbance (a case which may therefore provide useful indications on the validity and generality of such a model, especially if one considers that till date all existing theories have been successfully applied to the travelling-wave only).

The numerical simulations confirm the applicability of the resonance model to this specific case.

Extended regions exist at any time within the liquid bridge where the angular spin of the fluid is locally equal to the (as phenomenologically experienced by a fixed local observer) velocity of propagation of the hydrothermal disturbance in the azimuthal direction. As time passes most of particles stay attached to isosurfaces where the axial vorticity is close to zero (not limited to specific radial planes, but also including a surface of variable shape extended over $360^{\circ}$ ).

Just as in the case of PAS formed in the travelling-wave state, the emerging structures can be said to be an "illusion" as the particles do not really attach one another to form solid items and do not occupy fixed positions. All particles experience an endless motion. If a particle is located on an equilibrium meridian plane it performs a continuous revolution around the core of the toroidal Marangoni roll with some limited back-and-forth oscillations in the azimuthal direction. If it is not located on one of the six radial equilibrium sections, it is quickly driven back to one of such loci or it can "survive" for some time in the intermediate space provided it is captured by the circumferential isosurface where the axial vorticity is close to zero (once a particle is captured, if its axial coordinate changes, its radial and azimuthal coordinates change accordingly in such a way that it remains located in proximity to the isosurface). This process is very dynamic as this azimuthally extended surface is not stationary (as time passes, following the general pulsating behaviour of the termofluid-dynamic field, it changes shape and moves from the interior of the liquid bridge towards its external liquid/gas interface and then back towards the interior). By virtue of this mechanism, particles are transferred occasionally from an equilibrium plane to another.

Stripped to its basics, the related explanation for PAS formation envisions a restoring effect played by the vorticity-wave coupling mechanism in keeping particles confined to specific zones of the 
physical space, in particular, those where particles cannot phase lock with the travelling disturbance. Among other things, the resulting "equilibrium" model is susceptible of a remarkable analogy with the famous Chladni's series of experiments on patterns formed by sand on vibrating plates (sharing with the present situation many common elements), which further supports the physical relevance and generality of the proposed model.

There is no doubt that some of the theoretical arguments elaborated in this study would deserve a own exhaustive treatment and that the investigation of PAS in standing waves will attract additional future interest. As mentioned above, the work brought forward in this paper, was expressly undertaken to gain further understanding of the effectiveness of the resonance model in comparison with other theories. The fact that both experimental and numerical results discussed here perfectly fit the processes that one would expect to be operative under the influence of the resonance mechanism also in a standing-wave flow, suggests that phenomenon-controlling steps have been taken into account, and that the Schwabe-Pushkin theory may be indeed the correct one.

Among other things, the present study will also support the preparation of the microgravity experiment JEREMI (Japanese European Research Experiment on Marangoni Instabilities). Being developed by the "International Topical Team on Marangoni instabilities in systems with cylindrical symmetry" (with the support of JAXA and ESA) and scheduled for execution on board the International Space Station on 2016, this experiment will provide a relevant opportunity for investigating the dynamics of particle accumulation phenomena in liquid bridges.

The basic information gleaned from these future analyses will help scientists to develop better models to predict the properties of coherent particulate structures. Those, in turn, will lead toward ways to improve industrial technologies, increasing efficiency, reducing costs, or perhaps creating new processes. It is also obvious that, apart from the potential practical applications enabled by these studies, understanding the rich contextual information associated with the related evolution mechanisms will help us in using it to infer the general principles which drive complex patterns in a variety of other phenomena occurring in the physical world.

\section{ACKNOWLEDGMENTS}

This work is supported by the Italian Space Agency (ASI) in the framework of the JEREMI (Japanese and European Research Experiment on Marangoni Instabilities) Project (ASI contract $\mathrm{I} / 048 / 11 / 0$ ) for the preparation and execution of an experiment onboard the International Space Station (ISS).

\section{References}

[1] D. Langbein and W. Heide, The separation of liquids due to Marangoni convection, Adv. Space Res., 4(5), 27-36, (1984).

[2] B. Braun, C. Ikier, H. Klein and D. Woermann, Thermocapillary Migration of Droplets in a Binary Mixture with Miscibility Gap during Liquid/Liquid Phase Separation under Reduced Gravity, J. Colloid Interface Sci., 159(2), 515-516, (1993).

[3] L. Ratke, G. Korekt and S. Drees, Solidification of immiscible alloys, ESA SP 385, pag. 247, (1996).

[4] H. Gao, P.S. Ayyaswamy, and P. Ducheyne, Dynamics of a microcarrier particle in the simulated microgravity environment of a rotating wall vessel, Microgravity Sci Technol X, 154-165, (1997). 
[5] S.R. Pollack, D.F. Meaney, E.M. Levine, M. Litt and E.D. Johnston, Numerical Model and Experimental Validation of Microcarrier Motion in a Rotating Bioreactor, Tiss. Engin., 6, 519-530, (2000).

[6] C. F. M. Coimbra and M. H. Kobayashi, On the viscous motion of a small particle in a rotating cylinder, J. Fluid Mech. 469, 257-286, (2002).

[7] M. Lappa, C. Piccolo, Higher modes of Mixed Buoyant-Marangoni unstable convection originated from a droplet dissolving in a liquid/liquid system with miscibility gap, Phys. Fluids, 16(12), 4262-4272, (2004). M. Lappa, C. Piccolo, L. Carotenuto, Mixed buoyant-Marangoni convection due to dissolution of a droplet in a liquid-liquid system with miscibility gap, European Journal of Mechanics/B Fluids, 23/5, 781-794, (2004).

[8] M. Lappa, Assessment of VOF Strategies for the analysis of Marangoni Migration, Collisional Coagulation of Droplets and Thermal wake effects in Metal Alloys under Microgravity conditions, Computers, Materials \& Continua, 2(1), 51-64, (2005).

[9] M. Lappa, Oscillatory convective structures and solutal jets originated from discrete distributions of droplets in organic alloys with a miscibility gap, Phys. Fluids, 18 (4), 042105, (2006).

[10] E. Saeedi, S. Abbasi, K.F. Bohringer and B.A. Parviz, Molten-Alloy Driven Self-Assembly for Nano and Micro Scale System Integration, Fluid Dyn. Mater. Process., 2(4), 221-246, (2006).

[11] E.E.Michaelides, Review - The transient equation of motion for particles, bubbles, and droplets, J. Fluids Eng., 119, 233-247, (1997).

[12] E. Balkovsky, G. Falkovich, and A. Fouxon, Intermittent Distribution of Inertial Particles in Turbulent Flows, Phys. Rev. Lett. 86, 2790-2793, (2001).

[13] I. J. Benczik, Z. Toroczkai, and T. Tél, Selective Sensitivity of Open Chaotic Flows on Inertial Tracer Advection:Catching Particles with a Stick, Phys. Rev. Lett., 89 (16), 164501, (2002).

[14] T. Sapsis and G. Haller, Clustering criterion for inertial particles in two-dimensional timeperiodic and three-dimensional steady flows, Chaos, 20, 017515, (2010).

[15] D. Schwabe, P. Hintz, and S. Frank. New features of thermocapillary convection in floating zones revealed by tracer particle accumulation structures (PAS). Microgravity Sci. Technol., 9, 163-168, (1996). D. Schwabe, S. Tanaka, A. Mizev, and H. Kawamura. Particle accumulation structures in time-dependent thermocapillary flow in a liquid bridge under microgravity conditions. Microgravity Sci. Technol., 18, 117-127, (2006).

[16] D. Schwabe, A.I. Mizev, M. Udhayasankar and S. Tanaka, Formation of dynamic particle accumulation structures in oscillatory thermocapillary flow in liquid bridges, Phys. Fluids, 19(7), 072102, (2007) .

[17] I. Ueno, Y. Ono, D. Nagano, S. Tanaka and H. Kawamura (2000), Modal oscillatory structure and dynamic particle accumulation in liquid-bridge Marangoni convection, Proceedings of the Fourth JSME-KSME Thermal Engineering Conference, Kobe, Japan, 1-6 Oct. 2000, The Japan Society of Mechanical Engineers, Tokyo, Japan, 2000 Vol. 3, pp. 265-270. http://ci.nii.ac.jp/naid/110002497436/

[18] I. Ueno, Y. Abe, K. Noguchi, and H. Kawamura, Dynamic particle accumulation structure (PAS) in half-zone liquid bridge - Reconstruction of particle motion by 3-D PTV, Adv. Space Res., 41(12), 2145-2149 (2008).

[19] H.C. Kuhlmann, M. Lappa, D. Melnikov, R. Mukin, F.H. Muldoon, D. Pushkin, V.S. Shevtsova, and I. Ueno, The JEREMI-Project on thermocapillary convection in liquid bridges. Part A: Overview of Particle Accumulation Structures, Fluid Dyn. Mater. Process., 10 (1), 1-26 (2014) [20] D. Pushkin, D. Melnikov, V. Shevtsova, Ordering of Small Particles in One-Dimensional Coherent Structures by Time-Periodic Flows, Phys. Rev. Lett., 106, 234501 (2011).

[21] L. R. Huang, E. C. Cox, R. H. Austin, and J. C. Sturm, Continuous Particle Separation Through Deterministic Lateral Displacement, Science 304 (5673), 987-990, (2004). 
[22] M. Balvin, E. Sohn, T. Iracki, G. Drazer, and J. Frechette, Directional Locking and the Role of Irreversible Interactions in Deterministic Hydrodynamics Separations in Microfluidic Devices, Phys. Rev. Lett., 103, 078301, (2009)

[23] J. Herrmann, M. Karweit, and G. Drazer, (2009), Separation of suspended particles in microfluidic systems by directional-locking in periodic fields, Phys. Rev. E, 79, 061404, (2009).

[24] F. H. Muldoon and H. C. Kuhlmann. Coherent particulate structures by boundary interaction of small particles in confined periodic flows. Physica D, 253, 40-65, (2013)

[25] M. Lappa, On the Existence and Multiplicity of One-dimensional Solid Particle Attractors in Time-dependent Rayleigh-Bénard Convection, Chaos, 23(1), 013105, (2013).

[26] M. Lappa, Assessment of the role of axial vorticity in the formation of Particle Accumulation Structures in supercritical Marangoni and hybrid thermocapillary-rotation-driven flows, Phys. Fluids, 25(1), 012101, (2013).

[27] P. B. Rhines. Waves and turbulence on a beta-plane. J. Fluid Mech., 69, 417-443, (1975). M. Lappa, Rotating Thermal Flows in Natural and Industrial Processes (John Wiley \& Sons, Chichester, England, 2012).

[28] D. Schwabe and A.I. Mizev, Particles of different density in thermocapillary liquid bridges under the action of travelling and standing hydrothermal waves, Eur. Phys. J. Special Topics 192, 13-27, (2011).

[29] V. Shevtsova, A. Mialdun, H. Kawamura, I. Ueno, K. Nishino, M. Lappa, Onset of Hydrothermal Instability in Liquid Bridge. Experimental Benchmark, Fluid Dyn. Mater. Process., 7(1), 1-28, (2011).

[30] M. Lappa, R. Savino and R. Monti, Three-dimensional numerical simulation of Marangoni instabilities in liquid bridges: influence of geometrical aspect ratio, Int. J. Num. Meth. Fluids, 36 (1), 53-90, (2001).

[31] A. Babiano, J.H.E. Cartwright, O. Piro and A. Provenzale, Dynamics of a small neutrally buoyant sphere in a fluid and targeting in Hamiltonian systems, Phys. Rev. Lett., 84, 5764-5767, (2000).

[32] K. Boronska and L.S. Tuckermann, "Standing and travelling waves in cylindrical RayleighBénard convection", J. Fluid Mech., 559, 279-298 (2006).

[33] D. Melnikov, D. Pushkin, and V. Shevtsova, Accumulation of particles in time-dependent thermocapillary flow in a liquid bridge. Modeling of experiments, Eur. Phys. J. Special Topics, 192, 29-39, (2011).

[34] M. Lappa, On the variety of particle accumulation structures under the effect of gjitters, $J$. Fluid Mech., 726, 160-195, (2013).

[35] M. Lappa, Three-dimensional numerical simulation of Marangoni flow instabilities in floating zones laterally heated by an equatorial ring, Phys. Fluids, 15(3), 776-789 (2003); Combined effect of volume and gravity on the three-dimensional flow instability in non-cylindrical floating zones heated by an equatorial ring, Phys. Fluids, 16(2), 331-343 (2004).

[36] M. Lappa, R. Savino, 3D analysis of crystal/melt interface shape and Marangoni flow instability in solidifying liquid bridges, Journal of Computational Physics, 180 (2), 751-754 (2002).

[37] M.Lappa, S. Yasushiro, N. Imaishi, 3D numerical simulation of on ground Marangoni flow instabilities in liquid bridges of low Prandtl number fluid, Int. J. Num. Meth. Heat Fluid Flow, 13 (3), 309-340, (2003).

[38] K.A. Atkinson, An Introduction to Numerical Analysis (2nd ed.), (John Wiley \& Sons, New York, 1989).

[39] T. D.Rossing, Chladni's Law for Vibrating Plates, American Journal of Physics, 50, 271-274 (1982)

[40] M. Lappa, Thermal Convection: Patterns, Evolution and Stability (John Wiley \& Sons, Chichester, England, 2010). 